\title{
Localization of the Phenotypically Varying P270 Protein on dsRNA Virus-positive and Negative Trichomonas vaginalis Isolates
}

\author{
JF Alderete* \\ School of Molecular Biosciences, College of Veterinary Medicine, Washington State University, Pullman, Washington, USA
}

*Corresponding author: JF Alderete, School of Molecular Biosciences, MC7520, College of Veterinary Medicine, Washington State University, Pullman, WA 99164, USA.

To Cite This Article: JF Alderete. Localization of the Phenotypically Varying P270 Protein on dsRNA Virus-positive and Negative Trichomonas vaginalis Isolates. Am J Biomed Sci \& Res. 2021 - 14(2). AJBSR.MS.ID.001978. DOI: 10.34297/AJBSR.2021.14.001978.

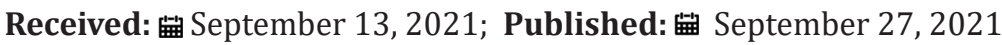

\begin{abstract}
Trichomonas vaginalis is a flagellated protist and causal agent for trichomonosis, the number one, nonviral sexually transmitted infection (STI). The parasites of all naturally-occurring isolates of T. vaginalis synthesize the immunogenic protein called P270, which is encoded by a single copy gene with numerous tandemly-repeated elements encoding the sequence DREGRD detected by the monoclonal antibody (MAb) C20A3. Isolates have been defined based on the absence (Type I) or presence (Type II) of T. vaginalis dsRNA virus (TVV). Type II TVV+ isolate organisms grown in batch culture have previously been shown to undergo phenotypic variation between surface and non-surface (cytoplasmic) expression of P270 using the MAb C20A3 in immunofluorescence (IF) assays. Type I TVV- trichomonads synthesize lower amounts of P270 that is only found in the cytoplasm. In this study both IF and immunoelectron microscopy (IEM) analysis were performed for the first time to localize P270 on and within both types of trichomonal isolates. IF experiments of Type II TVV+ parasites show whole surface labeling of intact, non-permeabilized organisms and surface and cytoplasmic fluorescence for permeabilized trichomonads. Immunocytochemistry of Type II trichomonads treated with MAb C20A3 presented gold-labeling of P270 throughout the external membrane as well as within peripheral vacuoles. Further, there was no P270 detected within the electron dense hydrogenosome organelles. On the other hand, for Type I TVV- permeabilized organisms, P270 is detected by IF only in the cytoplasm of some parasites, and immunocytochemistry confirmed that no trichomonads had surface P270. These data provide evidence for the first time of compartmentalization and surface localization of P270 among phenotypically varying, Type II TVV ${ }^{+}$. vaginalis compared to the Type I TVV organisms.
\end{abstract}

Keywords: dsRNA; Hydrogenosomes; MAb C20A3; Phenotypic variation; Immune-cytochemistry; Sexually transmitted infection; Trichomonas vaginalis ; Type I isolates; Type II isolates.

Abbreviations: dsRNA: double-stranded ribonucleic acid; FACS: fluorescence-activated cell sorting; h: hours; IEM: immune-electron microscopy; IF: immunofluorescence; min: minutes; MAb: monoclonal antibody; P270: 270-Kda protein; PBS: phosphate-buffered saline; PV: phenotypic variation; STI: sexually transmitted infection; TEM: transmission electron microscopy; T. vaginalis: Trichomonas vaginalis; Type II TVV+: dsRNA virus-harboring isolates; Type I TVV: dsRNA virus-minus isolates or progeny of virus-harboring isolates.

\section{Introduction}

Trichomonas vaginalis causes trichomonosis, the number one, non-viral STI, with adverse consequences to women's reproductive health $[1,2]$. One of the most immunogenic proteins of T. vaginalis is called P270 of $\sim 270-\mathrm{kDa}$ in size. This P270 protein has tandemly repeated immunogenic DREGRD-epitopes that are detected by the monoclonal antibody (MAb) C20A3 [3-7]. It was discovered by indirect immunofluorescence (IF) that some naturally-occurring isolates had subpopulations of parasites that were heterogeneous in the surface expression of P270 [4]. These isolates had both fluorescent and non-fluorescent organisms. Fluorescence activated cell sorting (FACS) [6] showed that 100 percent fluorescent trichomonads reflecting surface P270 became non-fluorescent. Likewise, sorted non-fluorescent parasites with cytoplasmic P270 reverted to fluorescent organisms with surface P270. It was hypothesized that parasite factors affect P270 synthesis and surface expression. Subsequently it was found that 
only T. vaginalis isolates infected with a dsRNA virus (called TVV) is related to the phenotypic variation of $\mathrm{P} 270[7,8]$, and the presence of TVV upregulated expression of the p270 gene concomitant with increased amounts of P270 transcripts and protein [8]. The presence of TVV and the property of phenotypic variation among some isolates further delineated two distinct isolate types among naturally-occurring clinical isolates [6]. Type I-designated isolates of T. vaginalis were $\mathrm{TVV}^{-}$and only expressed P270 cytoplasmically. Type II isolates were $\mathrm{TVV}^{+}$that had heterogeneous subpopulations of surface and non-surface-expressing organisms that underwent phenotypic variation [6].

Therefore, in this study it was important to visualize and provide immuno-cytochemical evidence on the role of TVV with respect to the surface localization of P270 among Type II $\mathrm{TVV}^{+}$ T. vaginalis parasites when compared with Type I TVV- isolate trichomonads. It was then found that long-term batch culture of $\mathrm{TVV}^{+}$isolate organisms resulted in the loss of TVV [6-8]. These isogenic $\mathrm{TVV}^{-}$progeny trichomonads were analyzed and compared to the original $\mathrm{TVV}^{+}$parental parasites [8]. Finally, as the function for P270 is unknown and no data exist regarding the intracellular location of P270, immuno-cytochemical localization experiments were performed. Among Type II $\mathrm{TVV}^{+}$organisms, P270 was seen throughout the external region of the membrane, in vesicles peripheral to the Golgi complex, and within vacuoles distinct from the electron dense hydrogenosomes involved in energy metabolism $[9,10]$. No surface P270 was detected among Type I $\mathrm{TVV}^{-}$trichomonads and P270 was detected in the cytoplasm within a subpopulation of organisms. These results reinforce important roles for Type II $\mathrm{TVV}^{+}$in the synthesis [8] and surface localization of P270.

\section{Methods}

\section{dsRNA virus and Trichomonas vaginalis}

The P270 phenotypically varying Trichomonas vaginalis 347 and T068-II are Type II TVV+ fresh clinical isolates, and T. vaginalis NYH 286 is a Type II TVV+ long-term grown laboratory isolate used previously [3-6,8, 11-19]. Growth of trichomonads in Trypticaseyeast extract-maltose (TYM) medium supplemented with $5 \%$ horse serum has been detailed earlier $[4,6,16]$. Extended batch culture of $\mathrm{TVV}^{+}$isolate 347 resulted in the loss of the dsRNA virus $\left(\mathrm{TVV}^{-}\right)$ and inability to surface express P270 [7]. Loss of dsRNA and virus particles was confirmed as detailed before $[3,7]$.

\section{Quantitation of P270 in trichomonads}

It was established that Type I $\mathrm{TVV}^{-}$isolate trichomonads synthesize lower amounts of P270 when compared to the phenotypically varying Type II $\mathrm{TVV}^{+}$isolate organisms $[3,7,8]$. Therefore, it was important to quantitate amounts of P270 among the three virus-harboring T. vaginalis isolates used in this study as well as $\mathrm{TVV}^{-}$trichomonads derived from isolate 347 that lost the dsRNA virus after extended batch cultivation. This quantitation would then permit establishing the amounts of P270 within trichomonads with the IF and immune-cytochemistry observations. The amounts of P270 were quantitated using an established previously published protocol [3]. Further, as an alternative method of quantitation, ultrathin sections of trichomonads collected in nickel grids as detailed below were treated with hybridoma supernatant with MAb C20A3 and secondary gold-conjugated antimouse IgG. One hundred randomly selected grids of Type II TVV 347 isolate organisms and of the $\mathrm{TVV}^{-}$progeny trichomonads were analyzed for the number of detectable P270.

\section{Immunofluorescence (IF) and monoclonal antibody (MAb) C20A3 detection of P270 on organisms}

Parasites grown overnight in TYM-serum medium were pelleted and washed in prewarmed $\left(37^{\circ} \mathrm{C}\right)$ phosphate-buffered saline (PBS), $\mathrm{pH} 7.2$, and fixed for 2 hours (h) with 4\% paraformaldehyde freshly prepared in $100 \mathrm{mM}$ sodium cacodylate buffer, $\mathrm{pH}$ 7.2. Fixed trichomonads were quenched using $50 \mathrm{mM}$ ammonium chloride containing 3\% bovine serum albumin (BSA). Washed parasites were placed on cover slips previously coated with poly-L-lysine, and bound organisms were incubated with hybridoma supernatant containing IgG $_{2 \mathrm{a}}$ MAb C20A3 [3-7]. Alternatively, prior to addition of MAb C20A3, duplicate cover slips with parasites were permeabilized with $0.1 \%$ Nonidet P-40 at RT and $100 \%$ acetone at $-20^{\circ} \mathrm{C}$. After incubation for 2-h, slides with fixed organisms were washed in PBS followed by addition of rodamine-conjugated goat antimouse IgG (Sigma Chemical Co., St. Louis, MO USA) diluted 1:100 in PBS and incubated for an additional 60 minutes (min). After washing in PBS, organisms were visualized with an Axiophot II Zeiss microscope with UV epifluorescence. Images were obtained with a Hamamatsuchilled CCD camera C5985. Negative controls included secondary gold-conjugated goat anti-mouse IgG, hybridoma supernatant containing an irrelevant MAb to Mycoplasma pneumoniae that is unreactive with trichomonad proteins, and hybridoma supernatant without antibody.

\section{Transmission electron microscopy (TEM)}

Organisms were first washed three times in PBS as above at $37^{\circ} \mathrm{C}$ prior to fixation overnight at RT in a solution of $0.4 \%$ glutaraldehyde, $4 \%$ paraformaldehyde, $0.5 \%$ sucrose, $1 \%$ picric acid, and $2 \mathrm{mM} \mathrm{CaCl}_{2}$ prepared in $100 \mathrm{mM}$ sodium cacodylate buffer. Fixed organisms were then washed 3 times over a 15-min period 
and dehydrated in ethanol prior to embedding in Unicryl (TedPella, Inc., Redding, CA, USA).

\section{Cryo-ultramicrotomy}

After overnight chemical fixation at $4^{\circ} \mathrm{C}$ with $4 \%$ paraformaldehyde and $0.1 \%$ glutaraldehyde in $0.1 \mathrm{M}$ cacodylate buffer, $\mathrm{pH} 7.2$, the specimens were infused in a mixture of $25 \%$ $(\mathrm{w} / \mathrm{v})$ polyvinylpyrrolidone (PVP) and 2.3M sucrose for 30min, submerged in liquid nitrogen and transferred to a cryoultramicrotome (Leica Ultracuts UCT). Cryosections were obtained at the temperature range of $-80^{\circ} \mathrm{C}$ to $-100^{\circ} \mathrm{C}$, collected on formvarcoated nickel grids and washed in PBS. This was followed by processing of the sections for treatment with MAb C20A3, as above. Finally, the grids containing cryosections were thinly embedded in a mixture of 9:1 (v:v) of 3\% polyvinyl alcohol and uranyl acetate [11] and observed in a JEOL 1210 electron microscope.

\section{Immuno-cytochemistry}

Ultrathin sections of trichomonads processed as above were collected in nickel grids and were quenched using $50 \mathrm{mM}$ ammonium chloride with 3\% BSA. The organisms were then washed in a PBS-2\% BSA buffer, pH 8.0, for 30-min. Grids were then incubated with MAb C20A3 hybridoma supernatant. After incubation for $3 \mathrm{~h}$ at RT, the grids were washed prior to addition of secondary $5 \mathrm{~nm}$ or $10 \mathrm{~nm}$ gold-conjugated goat antimouse IgG (Sigma). The grids were finally stained in the dark for 20-min at RT with $5 \%$ uranyl acetate followed by lead citrate. Sections were then visualized by TEM using a JEOL 1210 model microscope.

\section{Pre-embedding}

Pre-embedding of samples was performed to avoid loss of protein antigen detected by MAb C20A3. Parasites were fixed as above for TEM. After 3 washes in PBS, trichomonads were quenched for 30min using 50mM ammonium chloride with 3\% BSA and incubated with MAb for 3-h at RT under constant agitation. After several washes in PBS over a 30-min period, the organisms were incubated with $10 \mathrm{~nm}$ gold-conjugated goat anti-mouse antibody for $1 \mathrm{~h}$ at RT with constant stirring. The cells were then washed 3 times in PBS followed by re-fixation in $2.5 \%$ glutaraldehyde in $100 \mathrm{mM}$ sodium cacodylate buffer and routinely processed for TEM. Postfixation of parasites was in $1 \%$ osmium tetroxide and potassium ferricyanide, after which samples were dehydrated in acetone and embedded in Epon.

\section{Reproducibility}

Experiments on the NYH 286, T068-II and 347 T. vaginalis isolates were performed on at least four occasions under identical conditions and gave similar data.

\section{Results}

\section{Detection of P270 among dsRNA virus-positive and negative T, vaginalis isolates}

We first performed IF experiments with hybridoma supernatant containing MAb C20A3 on a Type II $\mathrm{TVV}^{+}$(virusharboring) phenotypically-varying T. vaginalis isolate 347 that was heterogeneous for surface and non-surface expressing P270 [6-8]. Figure 1 shows two separate experiments (panels a and b) using nonpermeabilized organisms with intense color imaging (column 1) and without color imaging (column 2) on the surface of representative organisms in the population expressing surface P270. In part c (columns 1 and 2) IF results using permeabilized trichomonads shows both surface and cytoplasmic labeling of P270 with Mab C20A3. The integrity of the parasites was evident by Nomarski differential interference contrast microscopy (column 3). Negative controls as indicated in Methods showed no immunoreactivity. Non-fluorescent trichomonads were identical to those unreactive with MAb as seen in Figure 2 below.

\section{Detection of P270 among $\mathrm{TVV}^{+}$T. vaginalis organisms and TVV progeny trichomonads.}

It has been established that Type II $\mathrm{TVV}^{+}$isolate organisms spontaneously abort the TVV during extended in vitro batch cultivation $[7,8]$. Comparative IF was performed using a parental surface-expressing P270 organism of isolate 347 with that of $\mathrm{TVV}^{-}$progeny trichomonads derived from the parental parasites. Figure 2 (panel a) shows the expected heterogeneous population of a $\mathrm{TVV}^{+}$isolate consisting of positive and negative surface P270 trichomonads [6,7]. Non-permeabilized $\mathrm{TVV}^{-}$progeny organisms gave $100 \%$ negative fluorescence for surface P270 equal to the negative, non-fluorescent trichomonads for part a. Therefore, IF was performed using permeabilized parasites, and not surprisingly, part b shows the detection of P270 for a single organism, which is consistent with previously established down-regulation of expression of P270 for $\mathrm{TVV}^{-}$trichomonads without surface P270, as shown below (Table 1) [3,8]. 


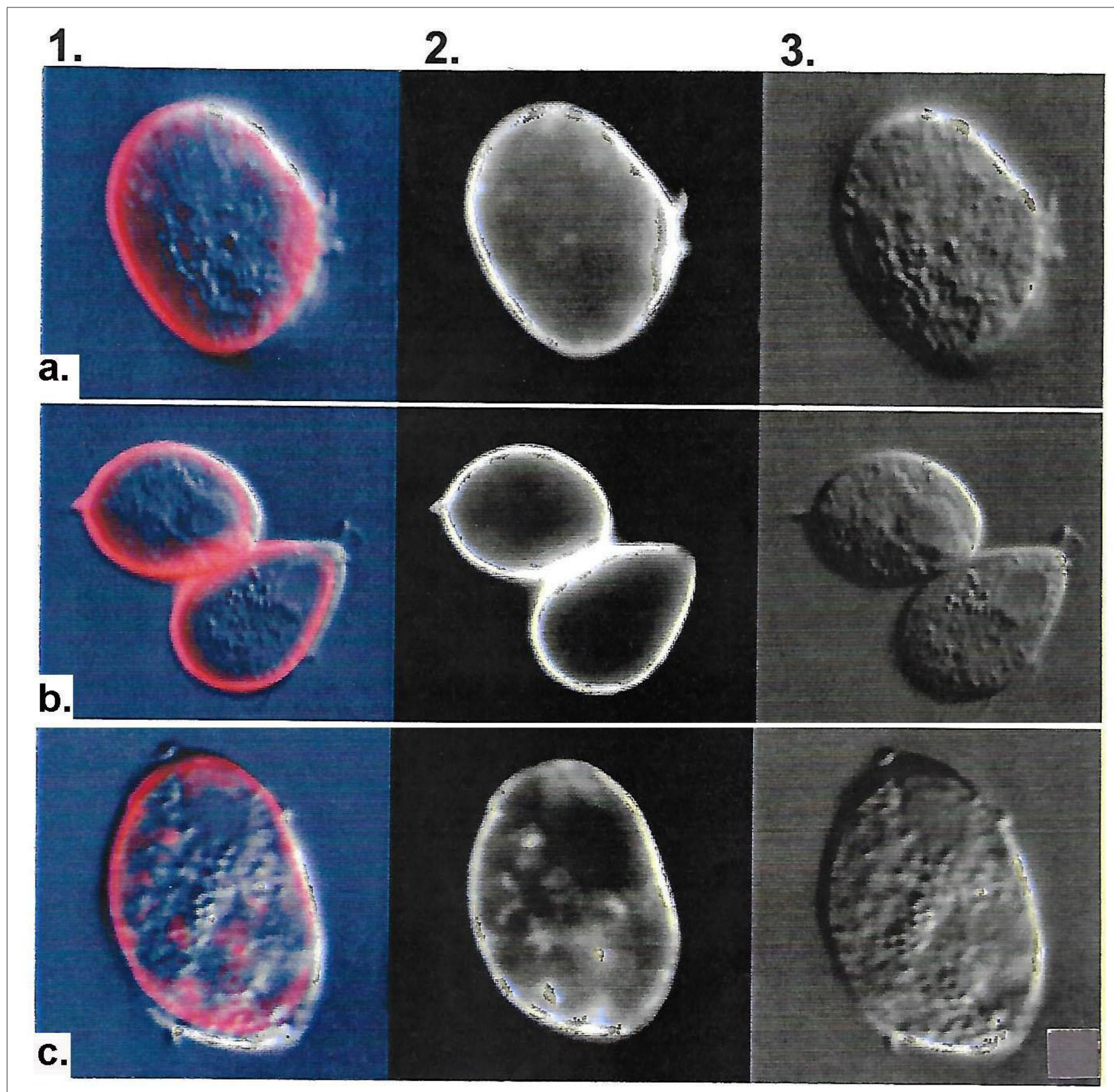

Figure 1: Representative immunofluorescence (IF) with IgG2a MAb C20A3 detection of surface P270 (panels a through c, columns 1 and 2) among Type II TVV+, dsRNA virus-harboring $T$. vaginalis isolate 347. Panels a and b were non-permeabilized organisms, and panel $\mathrm{c}$ were permeabilized parasites. Column 3 presents pictures of trichomonads visualized by Nomarsky differential interference microscopy to show the integrity of organisms during the experiments. Identical results were obtained with the other two TVV+ isolates NYH 286 and T068-II. Negative controls performed simultaneously included control hybridoma supernatant without antibody, secondary gold-conjugated anti-mouse lgG antibody alone, and irrelevant MAb of the same IgG2a to a Mycoplasma pneumoniae protein, which is unreactive with $T$. vaginalis. 


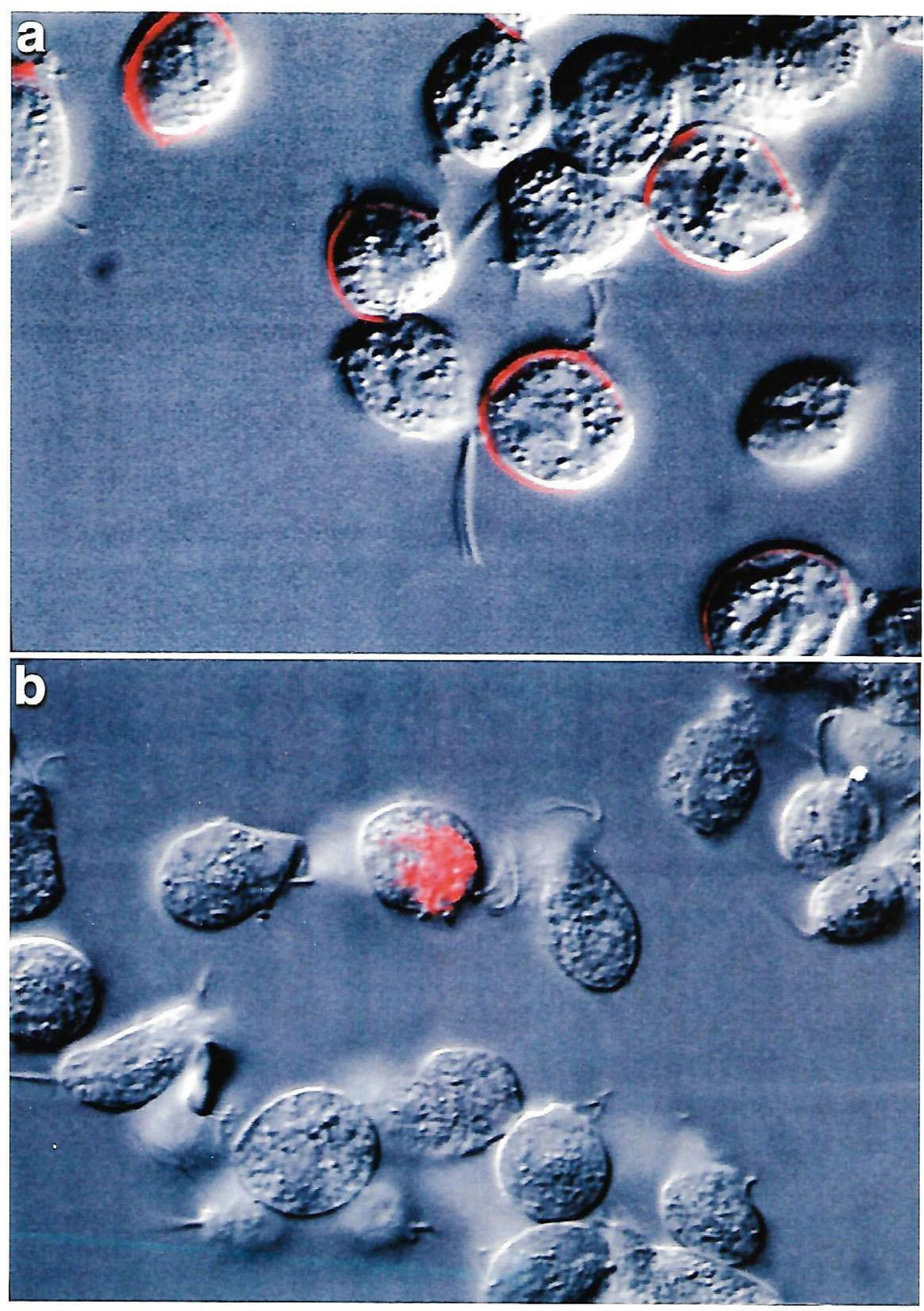

Figure 2: Comparative IF using MAb C20A3 to P270 as probe. Panel a shows non-permeabilized parental TVV ${ }^{+}$T. vaginalis isolate 347 with a heterogeneous population of surface versus non-surface P270-expressing trichomonads, as before [6]. The parasites without surface P270 synthesize lower amounts of P270 compared with surface expressing organisms [8]. Panel b shows the cytoplasmic P270 of a permeabilized progeny TVV- virus-negative 347 parasites derived from extended batch cultivation of the parental $\mathrm{TVV}^{+} 347$ isolate trichomonads. The virus-minus $T$. vaginalis isolates and progeny organisms have down-regulated expression of the p270 gene and synthesize lower amounts of P270 [6-8], which is also evidenced by Table 1 . The majority of the TVV-virus-negative 347 progeny organisms have no detectable P270 by IF. The same negative controls were used as in Figure 1 and gave no detectable P270. 
Table 1: Amounts of $\mathrm{P} 270$ protein among $T$. vaginalis isolates ${ }^{\mathrm{a}}$.

\begin{tabular}{|c|c|c|c|c|}
\hline \multirow{2}{*}{ Isolate } & \multirow{2}{*}{$\begin{array}{c}\text { dsRNA virus } \\
\text { type) }\end{array}$} & \multirow{2}{*}{$\begin{array}{c}\text { MAb C20A3 fluorescence } \\
\end{array}$} & & \multicolumn{2}{|c|}{$\boldsymbol{\mu g ~ p e r ~ 1 0 ~}^{\mathbf{7}}{\text { trichomonads } \pm \text { SD }^{\mathbf{d}}}^{\mathbf{c}}$} \\
\cline { 3 - 5 } & II & \pm & $4.33 \pm 0.05$ & $4.72 \pm 0.10$ \\
\hline NYH 286 & II & \pm & $4.28 \pm 0.10$ & $4.54 \pm 0.10$ \\
\hline T068-II & II & \pm & $7.22 \pm 0.04$ & $7.95 \pm 0.04$ \\
\hline 347 & I & - & $1.25 \pm 0.04$ & $1.07 \pm 0.1$ \\
\hline $347 \mathrm{v}^{\text {d }}$ & & experiment 1 & \\
\hline
\end{tabular}

a,bThe dsRNA virus was detectable by total nucleic extraction and ethidium bromide staining after agarose gel electrophoresis, as before $[7,8]$. Isolate type refers to Type II for presence of dsRNA virus and Type I for absence of the dsRNA virus among the fresh clinical isolates T068-II and 347 and the long-term grown NYH 286 isolate.

${ } \pm$ refers to the Type II virus-harboring isolate trichomonads comprised of heterogeneous fluorescent (P270 surface expressing) and non-fluorescent (non-surface P270) trichomonal populations based on mAb C20A3 reactivity with live organisms. The proportion of P270 surface-positive, fluorescent organisms versus P270 surface-negative non-fluorescent parasites is variable during batch culture for Type II isolate trichomonads. The - (minus) alone refers to the presence of a homogeneous population of non-fluorescent trichomonads based on indirect immunofluorescence with MAb C20A3 $[4,6,7]$. The procedure for fluorescence is that used previously by us $[4,6,7]$ and not the protocol in Methods for fixed trichomonads.

d $347 \mathrm{v}$ - refers to virus-minus progeny $T$. vaginalis organisms derived from the parental virus-positive isolate 347 after extended batch cultivation in medium.

\section{Quantitation of P270 among TVV+ T. vaginalis organisms and $\mathrm{TVV}^{-}$progeny trichomonads}

It was important to quantitate the amount of P270 among representative Type II $\mathrm{TVV}^{+}$isolate organisms compared to the progeny $\mathrm{TVV}^{-}$trichomonads. Table 1 shows that the $\mathrm{TVV}^{-}$progeny parasites synthesize only $\sim 20 \%$ the amount of P270 when compared to the $\mathrm{TVV}^{+}$isolate organisms of NYH 286, T068-II and 347. Further ultrathin sections with trichomonads embedded in nickel grids were then incubated with the MAb C20A3 to P270 and secondary $10 \mathrm{~nm}$ gold-conjugated secondary antibody to quantify the number of gold particles. In this experiment 100 grids of the Type II $\mathrm{TVV}^{+}$T. vaginalis isolate 347 were randomly selected and compared with an equal number of grids coated with the progeny $\mathrm{TVV}^{-}$trichomonads. For every 100 gold particles counted for the $\mathrm{TVV}^{+} 347$ isolate organisms, there was an average of 7 gold particles visualized for the progeny $\mathrm{TVV}^{-} 347$ parasites. This low number of 7\% P270 detected for progeny $\mathrm{TVV}^{-}$organisms when compared with $\mathrm{TVV}^{+}$parasites is consistent with the decreased amounts of P270 shown in Table 1 for the TVV 347 trichomonads. These experiments were performed on four separate occasions.

\section{Immuno-electron microscopy (IEM) localization of P270}

The P270 protein was then visualized on the long-term grown $\mathrm{TVV}^{+}$T. vaginalis isolate NYH 286 organisms that were expressing surface P270. The property of phenotypic variation and heterogeneous populations with fluorescent and non-fluorescent trichomonads using the MAb C20A3 was first observed with this isolate [4]. As can be seen in Figure 3 (panels a through c) from three different experiments, IEM on cryosections with trichomonads on nickel grids using the MAb C20A3 followed by gold-conjugated goat anti-mouse IgG showed strong labeling on the surface (panels a and b) and on the flagellum (panel c). Interestingly, for this $\mathrm{TVV}^{+} \mathrm{NYH}$ 286 isolate there was only some readily visible cytoplasmic labeling of P270. Not surprisingly, surface-labeling of P270 was also seen with the T068-II and $347 \mathrm{TVV}^{+}$isolate trichomonads. Equally noteworthy, no labeling was observed using negative controls (Methods).

In contrast, IEM was performed using the fresh clinical $\mathrm{TVV}^{+}$ T068-II isolate. Figure 4 \{panels a and b), in addition to surface labeling as mentioned above, intracellular immunogold-labeling was found in structures resembling coated pits (panel a) and withing vacuolar luminal membranes (panels b and c). No labeling was ever observed within the electron dense hydrogenosome organelle (panel c, labeled $\mathrm{H}$ ), which served as an internal negative control. As with prior experiments, there was no labeling with negative controls. Finally, Figure 5 (panels a and b) presents IEM of T. vaginalis isolate 347 and shows $\mathrm{P} 270$ on the surface and in elongated vesicles (labeled V) close to the trichomonal membrane. P270 was also detected within vacuolar luminal membranes and its contents (panel b), as seen above for isolate T068-II (Figure 4, panel a). Again, showing specificity in labeling, no gold particles were ever detected in the electron dense hydrogenosome seen on the left side of a clear elongated vacuole (panel b). As above, no gold particles were detected with the negative controls. 
a
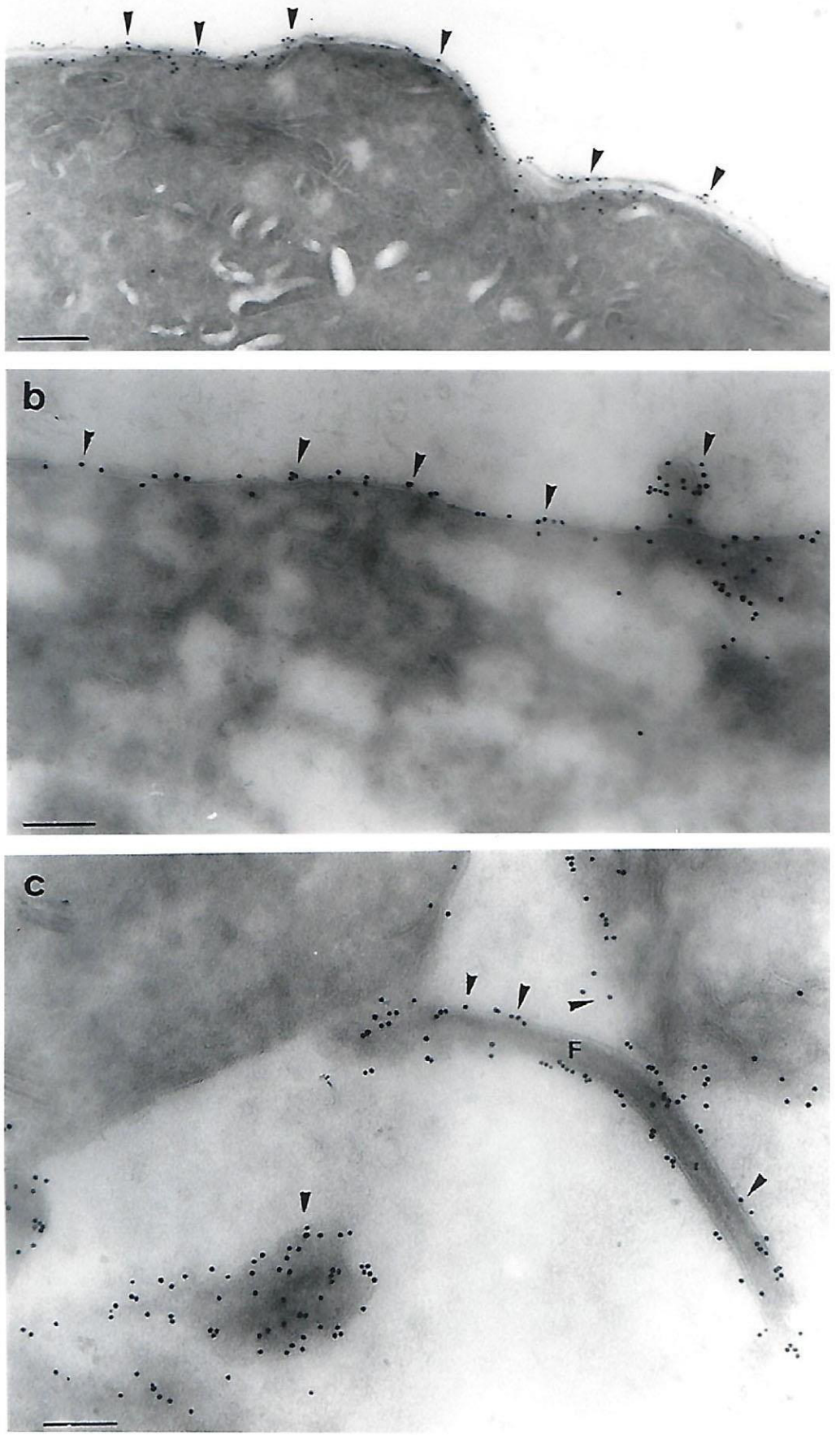

Figure 3: Immunoelectron microscopy (IEM) detection of P270 on cryosections on the surface of the Type II TVV ${ }^{+} T$. vaginalis isolate NYH 286. Strong surface labeling with MAb C20A3 and secondary gold-conjugated anti-mouse lgG was evident on three separate preparations from different experiments (panels a through c). The magnification for panel a was 52,500 times (bar $=0.19 \mu \mathrm{m}$ ), for panel b was 78,750 times $(\mathrm{bar}=0.14 \mu \mathrm{m})$ and for panel $c$ was 75,000 times $(c=0.13 \mu \mathrm{m})$. In panel $b$, P270 was detected in a small protrusion, and in panel c, P270 was readily detected on the flagellum (labeled F). No labeling was detected using the negative controls as for Figure 1. 

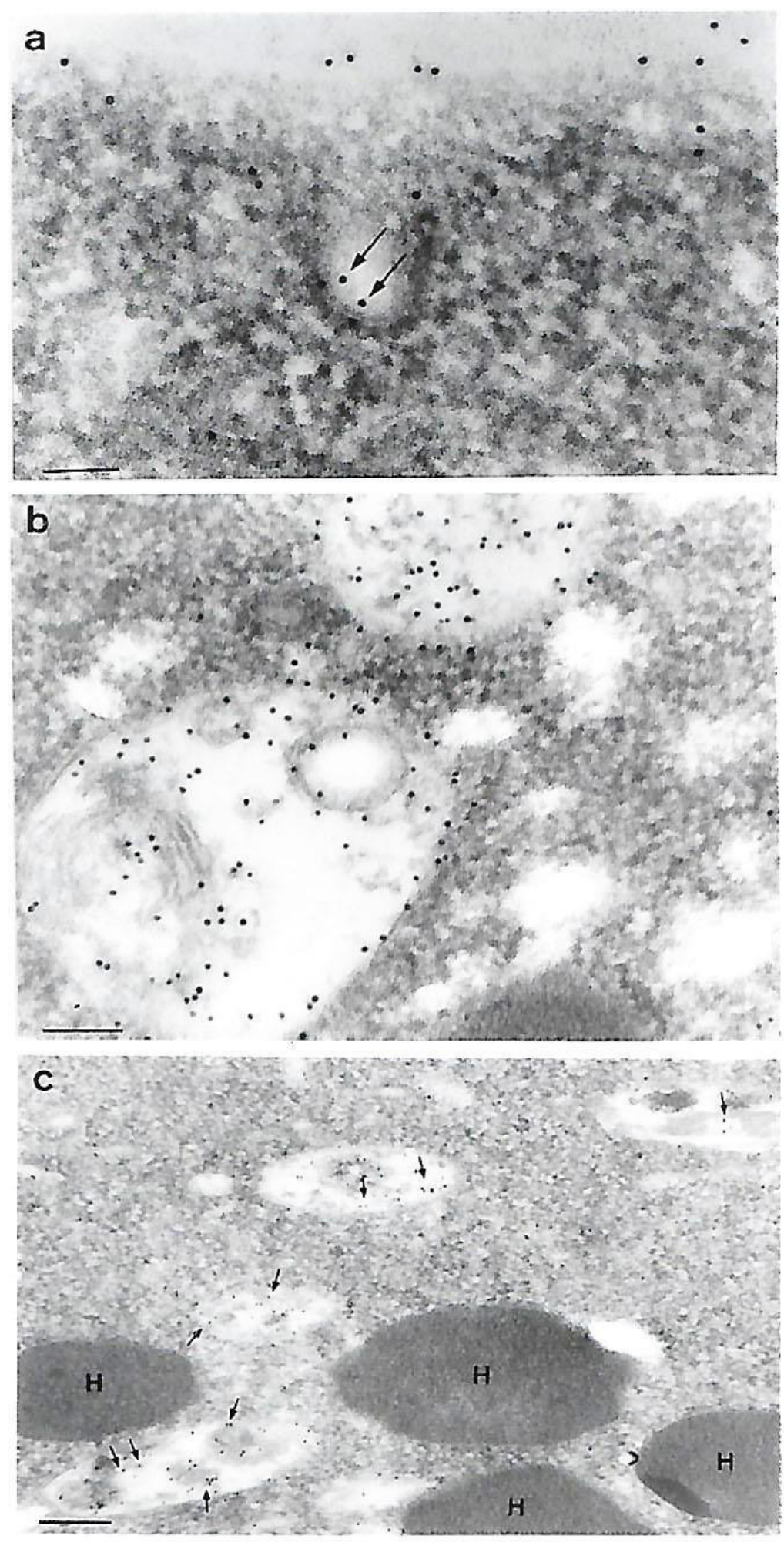

Figure 4: IEM detection of P270 (arrows) on the surface (panel a) and at various sites intracellularly (panels a through c) for the Type II TVV ${ }^{+} T$. vaginalis isolate T068-II. Panel a shows P270 labeling within a putative coated pit near the surface. Panel b shows extensive labeling of P270 within vacuoles, and panel $\mathrm{c}$ illustrates the absence of P270 within the double membrane, electron dense hydrogenosome organelle in contrast to the labeling in the surrounding vacuoles (arrows). The magnification for panel a was 120,000 (bar $=0.178 \mu \mathrm{m}$ ), for panel b was 96,000 (bar $=0.104 \mu \mathrm{m}$ ), and for panel $\mathrm{c}$ was 36,000 (bar $=0.28 \mu \mathrm{m})$. No labeling was detected using the negative controls as for Figure 1 . 


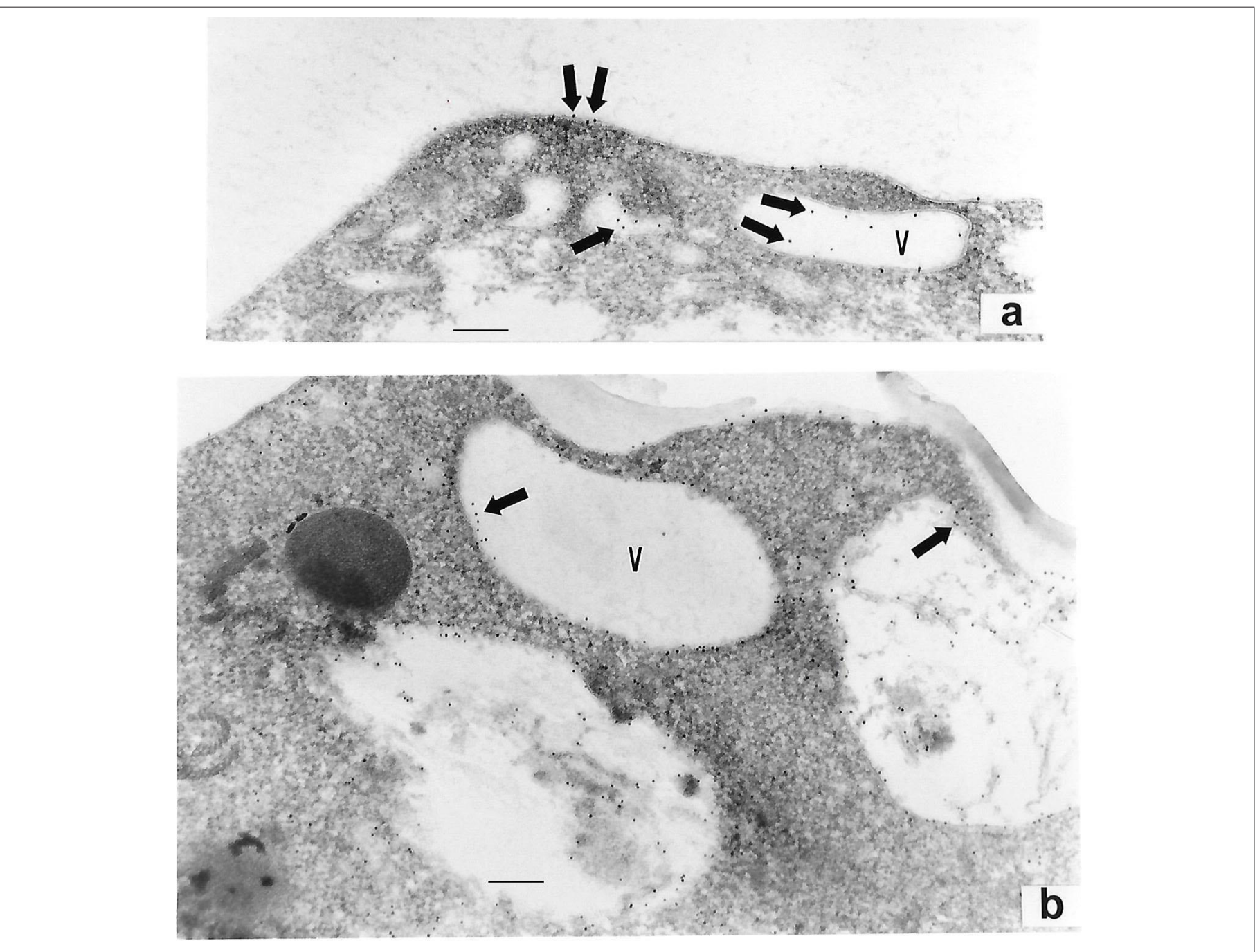

Figure 5: IEM detection of P270 with MAb C20A3 (arrows) on the surface (panel a) and at various intracellular vacuoles (labeled V) (panels a and b) for the Type II TVV+ $T$. vaginalis isolate 347 . Magnification for panel a was 67,5000 (bar $=0.148 \mu \mathrm{m}$ ) and for panel $\mathrm{b}$ was $56,000(\mathrm{bar}=0.178 \mu \mathrm{m})$. Here too there was no gold labeling within the electron dense hydrogenosome organelle shown to the left of panel b. No labeling was detected using the negative controls as for Figure 1.

\section{Discussion}

Evidence has shown that P270 is a member of a repertoire of high Mr protein immunogens that undergo coordinated phenotypic variation among trichomonads of T. vaginalis isolates that harbor a dsRNA virus (TVV) $[6,7,16]$. This property of phenotypic variation was originally discovered by FACS analyses and the experimental demonstration that trichomonads without surface P270 reverted to surface-positive for P270 during batch culture [6]. Similarly, purified parasites with surface P270 gave rise to a subpopulation of organisms without surface P270 [6]. The existence of a dsRNA virus within some $T$. vaginalis isolates was then used as a basis for defining two naturally-occurring types of trichomonal clinical isolates [6]. Type I TVV ${ }^{-}$(dsRNA virus negative) trichomonads were incapable of phenotypic variation and had decreased amounts of p270 mRNA and P270 protein. The Type II $\mathrm{TVV}^{+}$ (virus-harboring) isolate organisms both expressed P270 on the surface and underwent phenotypic variation that yielded heterogeneous populations of both surface and non-surface P270 by IF and immune-cytochemistry experiments (Figures 2-5). Both isolate types synthesize P270 [3], albeit Type II $\mathrm{TVV}^{+}$parasites have up-regulated expression of the p270 gene concomitant with higher amounts of P270 (Table 1) [3,8]. Among TVV trichomonad progeny derived from parental $\mathrm{TVV}^{+}$T. vaginalis 347 isolates, goldlabeled P270 was difficult to detect in the cytoplasm of nickel grids incubated with MAb C20A3, and no trichomonads had surface P270 by IEM.

The fact that clinical isolates and extended batch cultivation of trichomonads without P270 have never been discovered suggests an important role for this protein to the biology and survival of the organism. This study provides independent confirmation by 
immuno-cytochemical means of the surface expression and higher amounts of P270 among Type II $\mathrm{TVV}^{+}$isolate organisms. The existence of heterogeneous populations of trichomonads with and without surface P270 was demonstrated by immunoreactivity with MAb C20A3 (Figure 2), as before [4]. It is noteworthy that the MAb is directed to the DREGRD-epitope element that is tandemly repeated within P270 regardless of the presence or absence of TVV among isolates $[5,13,14]$. Earlier work on the molecular biology of P270 revealed a 17 amino acid transmembrane domain at the carboxy terminus $[5,13,14]$, showing why MAb C20A3 strongly detects P270 by interacting with the exposed and high copy number of DREGRD repeats for the P270 surface proteins. Further, the first amino acids at the amino-terminus of P270 have similarity to the putative signal sequence [17-20].

Importantly, immuno-cytochemical results were similar for the NYH 286, T068-II and 347 Type II TVV+ T. vaginalis isolates. The parasite membrane was readily labeled with MAb C20A3 (Figures 1 through 5). These data are also consistent with the known strong agglutination of surface-expressing parasites with MAb C20A3 [5]. This is the first time that P270 was detected on the flagella by IEM (Figure 3, panel c), and the absence of fluorescence of the flagella by IF may reflect the brightness of the surface relative to the thin structure of the flagella, whereas by IEM all structures are equally visualized. Interestingly, P270 was also localized in numerous cellular compartments, such as the peripheral vesicles and within vacuolar luminal membranes and its contents. The lack of hydrogenosome $[9,10]$ detection of P270 indicated specific localization to other compartments. That P270 was detected within structures resembling coated pits (Figure 4, panel a) may suggest a mechanism for surface targeting heretofore unknown for T. vaginalis. Finally, as it has been shown that the MAb C20A3 is cytotoxic to trichomonads with surface P270 [15], the property of phenotypic variation for the Type II TVV+ isolate organisms may represent an immune evasion strategy.

These findings on the immuno-localization of P270 contribute to our understanding of this unique and important protein of T. vaginalis organisms. I hope that this approach and results will provide new information that may shed light regarding the function of the molecule. Clearly, these studies establish a knowledge base that will be important for the future when compartmentalization analysis of P270 modified through alteration of the $p 270$ gene will be performed.

\section{Conclusion}

Visualization of the immunogenic P270 on the surface and within internal structures of T. vaginalis by IEM is an important step forward toward future understanding of the protein amino acid sequences mediating the pathways for compartmentalization and surface placement of P270. The isolation of progeny $\mathrm{TVV}^{-}$(dsRNA virus negative) organisms through batch culture of parental $\mathrm{TVV}^{+}$ parasites suggest these progeny are isogenic cultures that may be used for comparative analyses. Given that $\mathrm{TVV}^{-}$progeny organisms are equivalent to the Type I $\mathrm{TVV}^{-}$naturally-occurring isolate organisms is a significant observation that emphasizes the role of the dsRNA virus in the regulation of P270 gene expression, and possibly other T. vaginalis genes.

\section{Acknowledgement}

This study was supported by Public Health Service grants to JFA AI-45429 from the National Institute of Allergy and Infectious Diseases of the National Institutes of Health. The technical assistance of Leo Chang of the JFA laboratory and of Anna Lazzell of the Institutional Hybridoma Facility of the U. Texas Health Science Center atSan Antonio is especially acknowledged. As per instructions by the International Committee of Medical Journal Editors on "Who is an Author?" (http://www.icmje.org/recommendations/ browse/rolesand-responsibillities/defining-the-role-of-authorsand-contributors.html), I want to acknowledge Marlene Benchimol (MB) and members of her laboratory, including Natalia Lima Prates and Rafael Meyer Mariante who generated the electron microscopy pictures while at the Universidade Santa Ursula, Rio de Janeiro, Brazil. The technical assistance of Rívea Cristina Custódio Rodrigues of the laboratory was greatly appreciated. Financial support for MB was from Financiadora de Estudos e Projetos, Conselho Nacional de Desenvolvimento Cientifico e Tecnológico, Programa de Núcleos de Excelência, Fundação Carlos Chagas Filho de Amparo à Pesquisa do Estado do Rio de Janeiro, Coordenação de Aperfeiçoamento de Pessoal de Nivel Superior, and Associação Universitária Santa Úrsula.

\section{Conflict of Interest}

The author declares no conflicts of interest. The author alone designed the study and was responsible in the analyses and interpretation of data; in the writing of the manuscript, and in the decision to publish the results.

\section{References}

1. World Health Organization (1995). An overview of selected curable sexually transmitted diseases. WHO Global Programme on AIDS Report.

2. Hobbs MM, Sena Ac, Swygard H, Schwebke Jr (2008) Trichomonas vaginalis and trichomoniasis. Holmes KK, Sparling PF, Stamm WE, Piot P, Wasserheit JN, et al. (Ed). Sexually Transmitted Diseases, McGraw-Hill Medical, New York, USA.

3. Alderete JF, Neale KA (1989) Relatedness of structures of a major immunogen in Trichomonas vaginalis isolates. Infect Immun 57(6): 1849-1853.

4. Alderete JF, Suprun Brown L, Kasmala L (1986) Monoclonal antibody to a major surface glycoprotein immunogen differentiates isolates and subpopulations of Trichomonas vaginalis. Infect Immun 52(1): 70-75. 
5. Dailey DC, Alderete JF (1991) The phenotypically variable surface protein of Trichomonas vaginalis has a single, tandemly repeated immunodominant epitope. Infect Immun 59(6): 2083-2088.

6. Alderete JF, Kasmala L, Metcalfe E, Garza GE (1986) Phenotypic variation and diversity among Trichomonas vaginalis isolates and correlation of phenotype with trichomonal virulence determinants. Infect Immun 53(2): 285-293.

7. Wang A, Wang CC, Alderete JF (1987) Trichomonas vaginalis phenotypic variation occurs only among trichomonads infected with the doublestranded RNA virus. J Exp Med 166(1): 142-150.

8. Khoshnan A, Alderete JF (1994) Trichomonas vaginalis with a doublestranded RNA virus has upregulated levels of phenotypically variable immunogen mRNA. J Virol 68(6): 4035-4038.

9. Kulda J (1999) Trichomonads, hydrogenosomes and drug resistance. Int J Parasitol 29(2): 199-212.

10. Müller M (1992) Energy metabolism of ancestral eukaryotes: A hypothesis based on the biochemistry of amitochondriate parasitic protists. Biosystems 28(1-3): 33-40.

11. Tokuyasu KT (1986) Cryosections for immunohistochemistry. Proc $11^{\text {th }}$ Intl Congr EM. Kyoto, Japan, 42-43.

12. Alderete JF, Demes P, Gombosova A, Valent M, Janoska A, et al. (1987) Phenotypes and protein-epitope phenotypic variation among fresh isolates of Trichomonas vaginalis. Infect Immun 55(5): 1037-1041.

13. Musatovova O, Alderete JF (1998) Molecular analysis of the gene encoding the immunodominant phenotypically varying $\mathrm{p} 270$ protein of Trichomonas vaginalis. Microb Pathogen 24(4): 223-239.
14. Musatovova O, Alderete JF (1999) The Trichomonas vaginalis phenotypically varying p270 immunogen is highly conserved except for numbers of repeated elements. Microb Pathog 27(2): 93-104.

15. Alderete JF, Kasmala L (1986) Monoclonal antibody to a major glycoprotein immunogen mediates differential complementindependent lysis of Trichomonas vaginalis. Infect Immun 53(3): $697-$ 699.

16. Alderete JF (1987) Trichomonas vaginalis NYH 286 phenotypic variation may be coordinated for a repertoire of trichomonad surface immunogens. Infect and Immun 55(9): 1957-1962.

17. Alderete JF, O Brien JL, Arroyo R, Engbring JA, Musatovova O, et al. (1995) Cloning and molecular characterization of two genes encoding adhesion proteins involved in Trichomonas vaginalis cytoadherence. Molec Microbiol 17(1): 69-83.

18. Alderete JF, Engbring E, Lauriano C, O Brien (1998) Only two of the Trichomonas vaginalis triplet AP51 adhesins are regulated by iron. Microb Pathog 24(1): 1-16.

19. O Brien JL, Lauriano CM, Alderete JF (1996) Molecular characterization of a third malic enzyme-like AP65 adhesin gene of Trichomonas vaginalis. Microb Pathog 20(6): 335-349.

20. Lahti CJ, D Oliveira, Johnson PJ (1992) $\beta$-succinyl-coenzyme A synthetase from Trichomonas vaginalis is a soluble hydrogenosomal protein with an amino-terminal sequence that resembles mitochondrial presequences. J Bacteriol 174(21): 6822-6830. 